\title{
Numerical Solitons of Generalized Korteweg-de Vries Equations
}

\author{
Houde Han and Zhenli Xu \\ Department of Mathematics, University of Science and Technology of China, Hefei, \\ Anhui, 230026, P. R. China \\ E-mail: hhan@math.tsinghua.edu.cn(H. Han); xuzl@ustc.edu(Z.Xu)
}

\begin{abstract}
We propose a numerical method for finding solitary wave solutions of generalized Korteweg-de Vries equations by solving the nonlinear eigenvalue problem on an unbounded domain. The artificial boundary conditions are obtained to make the domain finite. We specially discuss the soliton solutions of the $K(m, n)$ equation and $\mathrm{KdV}-K(m, n)$ equation. Furthermore for the mixed models of linear and nonlinear dispersion, the collision behaviors of soliton-soliton and soliton-antisoliton are observed.
\end{abstract}

PACS numbers: 52.35.Sb, 05.45.Yv, 47.35.+i 


\section{Introduction}

In this paper, we propose a numerical method for finding solitary wave solutions, which we call numerical solitons, of generalized Korteweg-de Vries (GKdV) equations. The method is simple and effective to find numerical solitons and can thus be used to study the nonlinear mechanism of nonlinear evolution equations, especially in equations for which it is difficult to obtain soliton solutions by analytical tools. A sequence of GKdV equations which are not necessarily integrable are shown to admit soliton solutions by our method. In general, the GKdV equations are of the form

$$
u_{t}+G\left(u, u_{x}\right)_{x}+H(u)_{x x x}=0,
$$

where $G$ and $H$ are given functions. The $K(m, n)$ equation with $m, n \geq 1$,

$$
u_{t}+\left(u^{m}\right)_{x}+\left(u^{n}\right)_{x x x}=0,
$$

is a special case of the GKdV equation (11).

When $m=2, n=1$, it is the first important model equation, the Korteweg-de Vries $(\mathrm{KdV})$ equation,

$$
u_{t}+\left(u^{2}\right)_{x}+u_{x x x}=0
$$

which is integrable and admits sech ${ }^{2}$ soliton solutions. For the KdV equation (3), the linear dispersion and the nonlinear convection work against each other and exactly balance to result in a stable solution. Since Zabusky and Kruskal [1] numerically studied the elastic collisions of KdV solitons, the research of solitons has attracted many workers' attentions and interests.

For $n>1$, the $K(m, n)$ equation(2) is fully nonlinear [2]. Their soliton solutions have compact support and called compactons. For $m=n$, they assume a very simple form [2], namely

$$
u=\left\{\frac{2 \lambda n}{n+1} \cos ^{2}\left[\frac{n-1}{2 n}(x-\lambda t)\right]\right\}^{\frac{1}{n-1}}, \text { for }|x-\lambda t| \leq \frac{n \pi}{n-1},
$$

and zero otherwise, where $\lambda$ is a constant which represents the velocity of the travelling wave. The $K(m, n)$ equation is not integrable [2,3]; however, the collisions of the compactons are almost elastic by numerical experiments [2]. This suggests that the elastic collisions are shared by many nonlinear equations, even though they are not integrable. Hence, it seems of great interest to observe more equations to further study their nonlinear mechanisms.

However, the analytic ways are limited for many equations, due to the complexity of the nonlinear properties. The aim of the proposed numerical method is to obtain soliton

solutions for these equations. Our approach is to transform the nonlinear eigenvalue problems on the unbounded domain deduced from the travelling wave solutions of the equations into bounded problems by imposing an artificial boundary condition. These problems can then be solved numerically in an efficient way.

The rest of the paper is organized as follows. In section 2, we discuss the numerical method. In section 3, some application examples are proposed by using the method. Conclusions are given in section 4 . 


\section{The method}

\section{1. $K(m, n)$ equations}

Although the method is suitable for wider classes of nonlinear evolution equations, here we introduce the idea by considering the solitary wave solutions of $K(m, n)$ equation (2) for $m, n \geq 1$. Suppose that

$$
u(x, t)=f(\xi)=f\left(x-\lambda_{1} t\right)
$$

is a travelling wave solution of the $K(m, n)$ equation. It is easy to see that $\lambda_{1}$ and $f(\xi)$ satisfy the nonlinear eigenvalue problem

$$
\begin{aligned}
& -\lambda_{1} f+f^{m}+\left(f^{n}\right)_{\xi \xi}=0, \\
& f(\xi) \rightarrow 0 \quad \text { as } \xi \rightarrow \pm \infty .
\end{aligned}
$$

In general, the spectrum is continuous, and if $f(\xi)$ is an eigenfunction corresponding to $\lambda_{1}$, then so is $f\left(\xi-\xi_{0}\right)$ for any $\xi_{0} \in R$. Hence, it is very difficult to solve (6), (7) numerically.

If, however, we prescribe the amplitude of $f$ and the location of its maximum, for example by setting

$$
\begin{aligned}
& f(0)=1, \\
& f^{\prime}(0)=0,
\end{aligned}
$$

then (6)-(9) represents a nonlinear boundary-value problem on $(-\infty, 0]$ and $[0, \infty)$. Moreover, if $\lambda_{1}$ and $f(\xi)$ is a solution of (6)-(9), then

$$
u(x, t)=A f(B(A)(x-\lambda(A) t)),
$$

is a solitary wave solution of equation $K(m, n)$, with amplitude $A>0$, and functions $B(A)$ and $\lambda(A)$ to be determined. Let $\eta=B(A)(x-\lambda(A) t)$. Substituting (10) into equation (2), and using equation (6), we obtain

$$
\begin{aligned}
& -A \lambda(A) f+A^{m} f^{m}+A^{n} B(A)^{2}\left(f^{n}\right)_{\eta \eta} \\
& \quad=\left(-A \lambda(A)+A^{m} \lambda_{1}\right) f+\left(A^{n} B(A)^{2}-A^{m}\right)\left(f^{n}\right)_{\eta \eta} .
\end{aligned}
$$

We set

$$
B(A)=A^{\frac{m-n}{2}}
$$

and

$$
\lambda(A)=\lambda_{1} A^{m} .
$$

We know that $u(x, t)$ is a solitary wave solution of $K(m, n)$ with amplitude $A$ and velocity $\lambda_{1} A^{m}$. Therefore, we only need to find $\lambda_{1}$ and $f(\xi)$, and this allows us to find the other solitary wave solutions of $K(m, n)$.

For the numerical solution of the problem (6)-(9) on the unbounded domain $(-\infty,+\infty)$, we only need to consider the problem on the domain $[0, \infty)$, for the reasons

of symmetry. We introduce an artificial boundary at the point $\xi=b>0$, where $b$ is 
large enough. We use the boundary condition $f(b)=0$ to reduce the problem to a problem on the bounded interval $[0, b]$ for $m>1, n>1$ :

Find $\lambda_{1} \in R$ and $f(\xi)$, such that

$$
\begin{aligned}
& -\lambda_{1} f+f^{m}+\left(f^{n}\right)_{\xi \xi}=0,0<\xi<b \\
& f(0)=1, \\
& f^{\prime}(0)=0, \\
& f(b)=0 .
\end{aligned}
$$

We solve the problem numerically by a finite difference method. Let $h=b / N$ with a positive integer $N, \xi_{i}=i h, i=0,1, \cdots, N$. Then the interval $[0, b]$ is divided into $N$ subintervals by

$$
0=\xi_{0}<\xi_{1}<\cdots<\xi_{N}=b .
$$

Using the second-order central difference, we obtain

$$
-\lambda_{1} f_{i}+f_{i}^{m}+\frac{f_{i+1}^{n}-2 f_{i}^{n}+f_{i-1}^{n}}{h^{2}}=0, \quad i=0,1, \cdots, N-1 .
$$

Due to the boundary conditions we have

$$
f_{-1}=f_{1}, \quad f_{0}=1, \quad f_{N}=0 .
$$

The problem (18) with boundary conditions (19) is a nonlinear algebraic system which can be solved by the Newton iteration method.

\section{2. $M K d V-K(m, n)$ equation}

The second model we consider here is the $\operatorname{mKdV}-K(m, n)$ equation

$$
u_{t}+\left(u^{m}\right)_{x}+\left(u+u^{n}\right)_{x x x}=0 .
$$

We also assume the travelling wave solution

$$
u(x, t)=f(\xi)=f(x-\lambda t) .
$$

Then we have

$$
-\lambda f_{\xi}+\left(f^{m}\right)_{\xi}+\left(f+f^{n}\right)_{\xi \xi \xi}=0,
$$

which can be integrated once to yield

$$
-\lambda f+f^{m}+\left(f+f^{n}\right)_{\xi \xi}=C
$$

where $C$ is an arbitrary constant. Since we are interested in a localized solution, we know that:

(i). $f(\xi) \rightarrow 0$, as $\xi \rightarrow \pm \infty$; therefore we have $C=0$ in equation (23);

(ii). $f(0)=A$ is an extremum, i.e., $f^{\prime}(0)=0$. 
Similarly, we obtain the following nonlinear problem on $[0, \infty)$ :

$$
\begin{aligned}
& -\lambda f+f^{m}+\left(f+f^{n}\right)_{\xi \xi}=0, \\
& f(\xi) \rightarrow 0 \quad \text { as } \xi \rightarrow+\infty, \\
& f(0)=A, \\
& f^{\prime}(0)=0 .
\end{aligned}
$$

We introduce the artificial boundary point $\xi=b>0$, with $b$ large enough, and $m, n>1$. On the interval $[b, \infty)$, the equation $(24)$ can be linearized to

$$
-\lambda f+f_{\xi \xi}=0, \quad \xi \in[b, \infty),
$$

which has two linearly independent solutions,

$$
e^{\sqrt{\lambda} \xi} \text { and } e^{-\sqrt{\lambda} \xi}
$$

By the condition $(25), f(\xi)=C e^{-\sqrt{\lambda} \xi}$ with $\lambda>0$, and

$$
f^{\prime}(\xi)=-\sqrt{\lambda} C e^{-\sqrt{\lambda} \xi}=-\sqrt{\lambda} f(\xi) .
$$

Then at $\xi=b$ we have the artificial boundary condition

$$
f^{\prime}(b)=-\sqrt{\lambda} f(b)
$$

Thus we have reduced the problem to a problem on the bounded interval $[0, b]$ :

$$
\begin{aligned}
& -\lambda f+f^{m}+\left(f+f^{n}\right)_{\xi \xi}=0,0<\xi<b \\
& f(0)=A, \\
& f^{\prime}(0)=0, \\
& f^{\prime}(b)=-\sqrt{\lambda} f(b),
\end{aligned}
$$

which can again be solved by the finite difference method.

\section{Numerical examples}

Example 1. Use the proposed method in Section II, numerical solitary wave solutions of the GKdV equation can be obtained. We first consider the $K(2,2)$ and $K(3,3)$ equations to test the efficiency of the method.

(a)

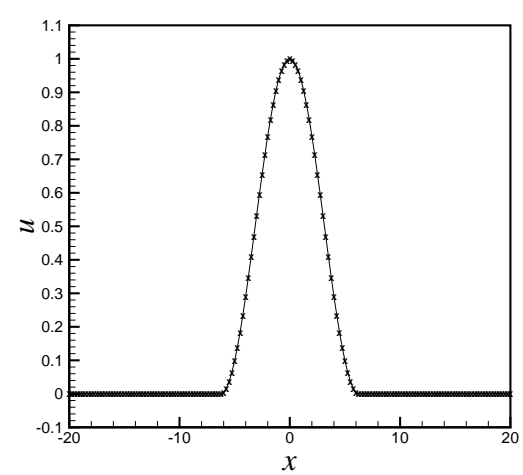

(b)

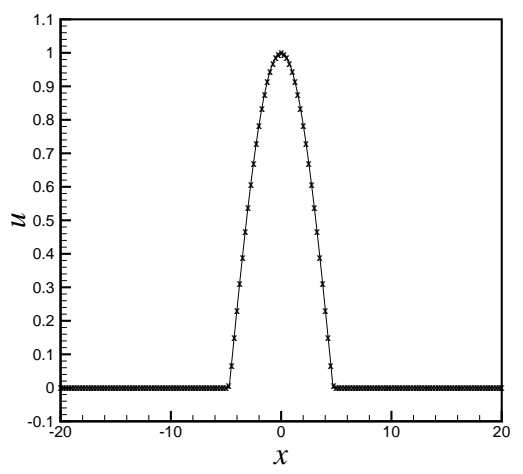

Fig. 1. Numerical solitons. Solid line is the exact solution. (a) $K(2,2)$; (b) $K(3,3)$. 
In this case, the Newton iteration maybe does not converge or the solution shows blowup phenomena with the grid refinement. To avoid these phenomena, a low pass filter has been used in iterate steps. Fig. 1 (a) and (b) show the numerical results with $h=0.25$. We see the numerical results are well agreed with the exact solutions.

Example 2. We show the numerical soliton to the $\operatorname{KdV}-K(2,2)$ equation

$$
u_{t}+\left(2 u^{2}\right)_{x}+\left(u+u^{2}\right)_{x x x}=0,
$$

which is a combination of the $\mathrm{KdV}$ and $K(2,2)$ equations. Unlike the $\mathrm{KdV}(3)$ and $K(m, n)$ (2) equations, it is a mixed dispersive model. Both linear and nonlinear dispersions are often presented in many physical applications, for example the CarmassaHolm equation [4] in water waves. It is interesting to solve them to study the interaction mechanism of linear and nonlinear structures [5].

Fig. 2(a) illustrates the shapes of three numerical solitons with amplitudes $A=2$, 1.5 and 1 , respectively. We also perform the numerical experiments of collisions with the three solitons which are located at the three isolated centers $x=-60,-30$ and 0 . Here, the fully implicit Crank-Nicolson scheme is used in the domain $[-80,80]$ with periodic boundary conditions. Fig. 2(b-d) shows the evolution of the three numerical solitons. Extensive tests all indicate that the collisions of the $\operatorname{KdV}-K(2,2)$ equation (34) are almost elastic.

(a)

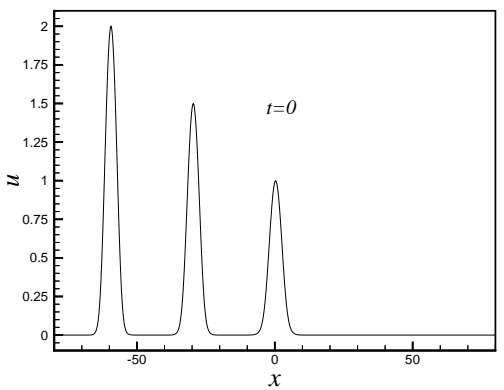

(c)

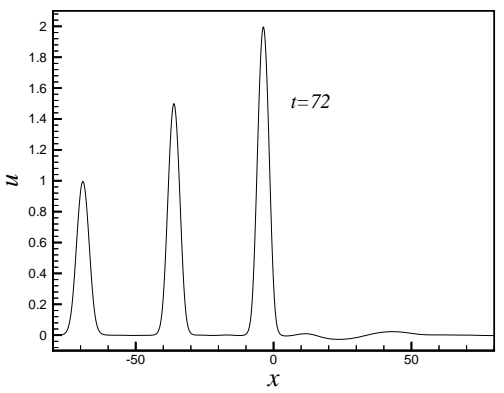

(b)

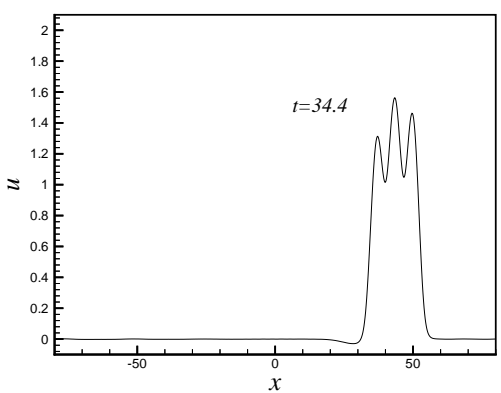

(d)

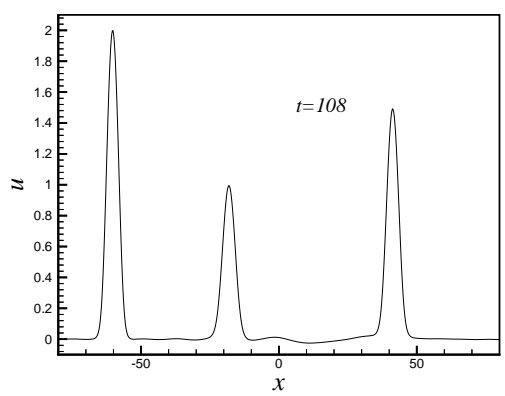

Fig. 2. The evolution of three numerical solitons.

Similarly to the behavior of the $K(2,2)$ compactons, the collisions of $\operatorname{KdV}-K(2,2)$ numerical solitons also create a small ripple (see Fig. 3). The amplitudes of the ripple are less than $3 \%$ of the amplitudes of all the solitons. However, their amplitudes do not almost decrease with the mesh is refined. The result illustrates that the creation of the collision ripple probably does not arise from the numerical accuracy but from its internal 
mechanism of the equation. Though this possibility appears reasonable, it needs to be confirmed by more proofs since now it has only been deduced from numerical results.

(a)

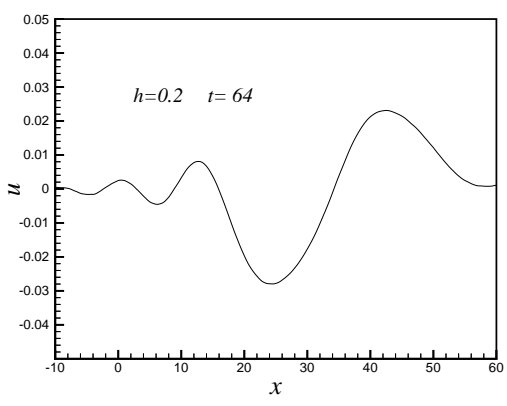

(b)

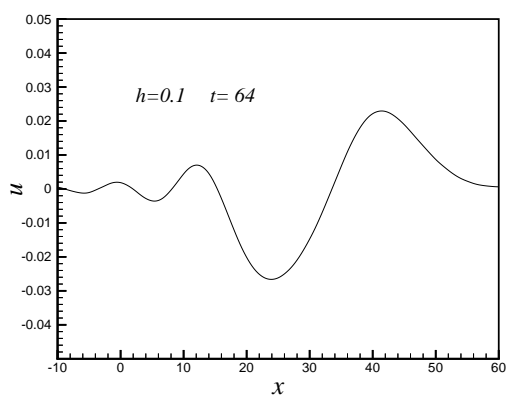

Fig. 3. The ripples created by the collision of solitons in different grids.

Example 3. The model equation we considered is called the $\operatorname{mKdV}-K(3,3)$ equation,

$$
u_{t}+\left(2 u^{3}\right)_{x}+\left(u+u^{3}\right)_{x x x}=0
$$

which is a combination of the modified $\mathrm{KdV}$ and $K(3,3)$ equations. Since all terms of the mKdV-K(3,3) equation (35) are odd, its soliton solutions are in pairs by the relationship $(u,-u)$. They propagate in the same direction with the same speed. The solitons with negative signs are normally called antisolitons.

The $K(2,2)$ equation also admits anticompactons which propagate in the opposite direction. However, it is difficult to numerically simulate compacton-anticompacton collisions because of instability. It is not clear whether or not the reason of this instability is due to numerical errors [2]. Thus, in order to better understand the reason, it is meaningful to first study the behavior of soliton-antisoliton collisions.

Fig. 4 shows the evolution of numerical soliton and antisoliton with amplitudes 2 and 1 , respectively. Here we take the spatial mesh size $h=0.025$ in $[-40,40]$ and periodic boundary conditions. We can see that their collisions are elastic if the tail oscillations originate from numerical in accuracies. In fact, extensive numerical experiments indicate that the oscillations decrease when the mesh is refined. This illustrates that the creation of oscillations are mainly caused by the numerical errors.

(a)

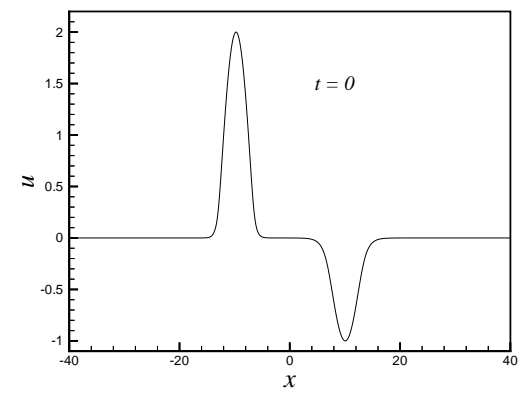

(b)

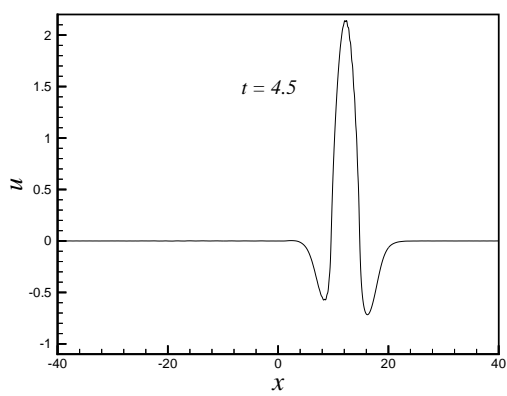


(c)

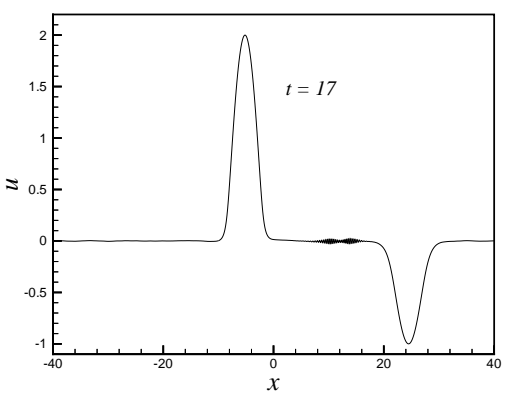

(d)

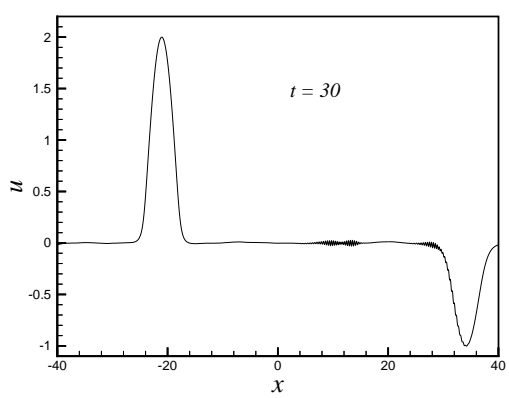

Fig. 4. The evolution of soliton-antisoliton collisions.

These oscillations are high-frequency waves which are caused by every collision because of the numerical errors. We see that the collisions of oscillations and solitons are also elastic (see Fig. 4(c)(d)). However, it is difficult to handle them unless we use a very fine grid size. This is also the reason why compacton-anticompacton collisions of the $K(m, n)$ equation are difficult to be simulated. Based on the above experiments, it is conjectured that the $K(m, n)$ compacton-anticompacton collisions are also elastic.

We know the Benjamin-Bona-Mahoney (BBM) equation

$$
u_{t}+\left(u^{2}\right)_{x}-u_{x x t}=0
$$

as a good example of a nonintegrable model that has also soliton and antisoliton solutions. Its soliton-soliton collisions are elastic, but numerical tests illustrate that its soliton-antisoliton collisions are inelastic [6]. Therefore, it is interesting to study what governs the behavior of solitons collisions, but we are still a long way from a good understanding of the wave mechanics [7].

\section{Conclusion}

In summary, we have reported a method to find numerical solitons of the GKdV equations which are not necessarily integrable. Using this method, we have given a basic study of soliton-soliton collisions of the $\operatorname{KdV}-K(2,2)$ equation and solitonantisoliton collisions of the mKdV-K(3,3) equation. This method and the numerical experiments will contribute to a future, more extensive study of the theoretical aspects of the nonlinear dispersive mechanism of evolution equations.

\section{Acknowledgments}

The authors wish to thank Professor H. Brunner for the valuable discussions. This work was supported by National Natural Science Foundations of China under Grant No. 10471073.

\section{References}

[1] N. J. Zabusky and M. D. Kruskal. Interaction of "Solitons" in a collisionless plasma and the recurrence of initial states. Phys. Rev. Lett., 15:240-243, 1965. 
[2] Philip Rosenau and James M. Hyman. Compactons: Solitons with finite wavelength. Phys. Rev. Lett., 70:564-567, 1993.

[3] P. Rosenau. On nonanalytic solitary waves formed by a nonlinear dispersion. Phys. Lett. A, 230:305-318, 1997.

[4] R. Camassa and D. Holm. An integrable shallow water equation with peaked solitons. Phys. Rev. Lett., 71:1661-1664, 1993.

[5] A. S. Fokas. On a class of physically important integrable equations. Physica D, 87:145-150, 1995.

[6] L. R. T. Gardner and G. A. Gardner. Solitary waves of the regularised long wave equation. J. Comput. Phys., 91:441-459, 1990.

[7] Y. A. Li, O. J. Olver, and P. Rosenau. Non-analytic solutions of nonlinear wave models. Preprint, 1998. 